\title{
INFRARED OBSERVATIONS OF THE $z=3.8$ RADIO GALAXY, 4C 41.17, WITH THE W. M. KECK TELESCOPE
}

\author{
James R. Graham, ${ }^{1,2}$ K. Matthews, ${ }^{3}$ B. T. Solfer, ${ }^{3}$ J. E. Nelson, ${ }^{1,4}$ W. Harrison, ${ }^{4}$ \\ J. G. Jernigan, ${ }^{5}$ S. Lin, ${ }^{3}$ G. Neugebauer, ${ }^{3}$ G. SMith,${ }^{4}$ and C. ZiomKowski ${ }^{3}$ \\ Received 1993 July 14 ; accepted 1993 October 12
}

\begin{abstract}
The near-infrared camera on the W. M. Keck telescope has been used to image the $z=3.8$ radio galaxy 4C 41.17. The $2 \mu \mathrm{m}$ continuum consists of a thin arc of emission aligned along the radio axis. There is close correlation between the optical, infrared, and radio emission. The color of $4 \mathrm{C} 41.17$ is blue $\left(R-K_{S}=2.7 \pm 0.1\right.$ mag). This may indicate ongoing star formation, but the origin of the continuum emission remains unclear. [O III] and $\mathrm{H} \beta$ emission makes a large contribution to the $K$-band flux. A number of faint red companions of 4C 41.17 are detected in the $2 \mu \mathrm{m}$ image. If these sources are at the same redshift as $4 \mathrm{C} 41.17$, then their red colors indicate ages of more than $\sim 0.5 \mathrm{Gyr}$ and an epoch of formation at $z \gtrsim 8$.

Subject headings: early universe - galaxies: active - galaxies: formation — radio continuum: galaxies
\end{abstract}

\section{INTRODUCTION}

Several galaxies with redshifts in the range 3-4 are now known. These galaxies are young, because for a wide range of cosmological parameters they have ages of less than $\sim 20 \%$ of the current age of the universe. Large telescopes allow detailed studies of distant galaxies, and therefore offer a window on the epoch of galaxy formation. Here we present some of the first observations made with the $10 \mathrm{~m} \mathrm{~W}$. M. Keck telescope on Mauna Kea, Hawaii: infrared imaging of the most distant known galaxy 4C 41.17, at $z=3.8$ (Chambers, Miley, \& van Breugel 1990).

$4 C 41.17$ is a steep spectrum radio galaxy, with a F-R II morphology. The central radio source is coincident with a large ( $\gtrsim 70 \mathrm{kpc}$ ) Ly $\alpha$ cloud. The Ly $\alpha$ and UV continuum are fairly well aligned with the radio emission. The galaxy is relatively bright at $2.2 \mu \mathrm{m}$, although little is known about the morphology (Chambers et al. 1990). High-resolution Hubble Space Telescope images of $4 \mathrm{C} 41.17$ show that the $R$-band emission is clumpy with a morphology that correlates with the radio on sub-kiloparsec scales (Miley et al. 1992).

\section{OBSERVATIONS AND REDUCTIONS}

4C 41.17 was observed on 1993 March 24 and 25 using the $10 \mathrm{~m} \mathrm{~W}$. M. Keck telescope with the facility near-infrared camera (Matthews et al. 1994). The camera is equipped with a SBRC $256 \times 256 \mathrm{InSb}$ array. The pixel size is $0 " 15$. We observed at $K(2.0-2.4 \mu \mathrm{m})$ and $K_{S}(2.0-2.3 \mu \mathrm{m})$, the former includes redshifted [O III] and $\mathrm{H} \beta$. An integration time of $21 \mathrm{~s}$ yields background-limited performance, while preventing saturation. Frames were obtained in groups of five and then the telescope was repositioned by a few arcseconds. Each frame was guided using an off-axis CCD camera. The exposure time for the central $40^{\prime \prime}$ of the resultant mosaic is $2372 \mathrm{~s}$ and $1386 \mathrm{~s}$ at $K_{s}$ and $K$, respectively.

\footnotetext{
${ }^{1}$ Astronomy Department, University of California, Berkeley, CA 94720.

${ }^{2}$ Alfred P. Sloan Research Fellow.

3 Palomar Observatory, California Institute of Technology, Pasadena, CA 91125.

${ }^{4}$ California Association for Research in Astronomy, 65-1120 Mamalahoa Highway, P.O. Box 220, Kamuela, HI 96743.

${ }^{5}$ Space Sciences Lab, University of California, Berkeley, CA 94720.
}

All frames were sky subtracted and then flat fielded using dark subtracted sky frames. The positions of two to three stars in each frame were used for spatial registration. Photometry was performed relative to UKIRT faint standards (Casali \& Hawarden 1992). The $K_{S}$ magnitudes of these stars are unknown. However, we chose blue standards, and adopted $K-K_{S}=0$ mag. The resultant photometric error should be less than 2\%. Figure 1 (Plate L2) is a finding chart for each object in the field, and Figure 2 is the color-magnitude diagram derived from the photometry listed in Table 1.

A 1200 s exposure $R$-band CCD image of $4 \mathrm{C} 41.17$, obtained at the $4 \mathrm{~m}$ telescope of the Kitt Peak National Observatory in 1989 March, was provided by Dickinson \& Spinrad (1993).

The positions of five stars present in the infrared and the $R$-band images were used to transform all the observations to a common coordinate frame. Astrometry of stars common to the Palomar Sky Survey and the $R$-band image permits us to establish absolute coordinates. The relative registration of the optical and infrared images is good to 0.1 , and the absolute coordinates are accurate to 0.5 .

\section{RESULTS}

The $K_{S}$ mosaic achieves a $1 \sigma$ limiting magnitude of 23.1 mag in a $3^{\prime \prime}$ diameter beam. The image size is seeing limited with a full width at half-maximum (FWHM) of 0 ".87. The $K$ mosaic limit is $22.7 \mathrm{mag}, 1 \sigma$, in the same-sized beam, and the FWHM is 0.65 . The $R$ images have $26.9 \mathrm{mag}$. $1 \sigma$ and $\mathrm{FWHM}=0$ ".9.

The $K_{S}$ image (Figs. 3 [Plate L3] and 4) shows that $4 \mathrm{C}$ 41.17 consists of a single nucleus at the center of a thin curved arc of emission that extends over $\simeq 6^{\prime \prime}$ or $26 \mathrm{kpc}\left(H_{0}=75 \mathrm{~km}\right.$ $\left.\mathrm{s}^{-1} \mathrm{Mpc}^{-1}, q_{0}=0.5\right)$. The arc is clumpy, a conclusion that is reinforced by a $K_{S}$ mosaic with FWHM of 0 ". 6 constructed by selecting 50 images with the best seeing. There is a marked concentration of faint $\left(K_{S} \simeq 20-23 \mathrm{mag}\right)$ objects near $4 \mathrm{C} 41.1$ ) of which only the brightest has been previously detected (object 12 is object $Z$ of Chambers et al. 1990). At least seven such objects $(2-5,9$, and 12$)$ are located within a radius of $12^{\prime \prime}$ $(53 \mathrm{kpc})$. All the objects are resolved.

Intrared spectra of $4 \mathrm{C} 41.17$ show that the $K_{S}$ filter band pass is free of bright emission lines (Eales \& Rawlings 1993), 
PLATE L2

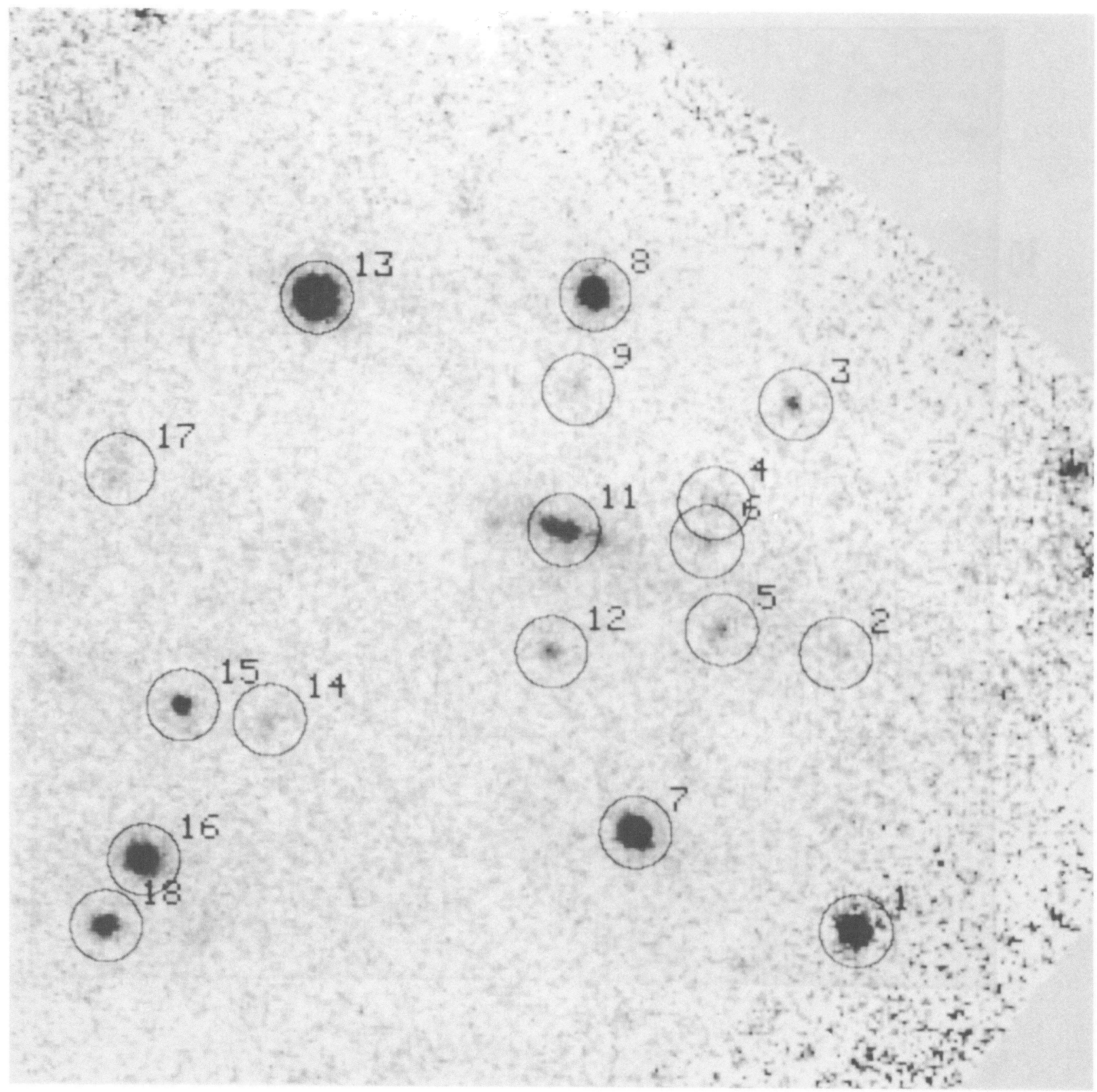

Fig. 1.-Finding chart for the objects shown in Fig. 2 overlaid on the $K$ image of $4 \mathrm{C} 41.17$ (object 11). The field of view is $\sim 45^{\prime \prime}$ on a side. North is at the top, and east to the left. The circles have a diameter of $3^{\prime \prime}$.

GraHAM et al. (see 420, L5) 
PLATE L3

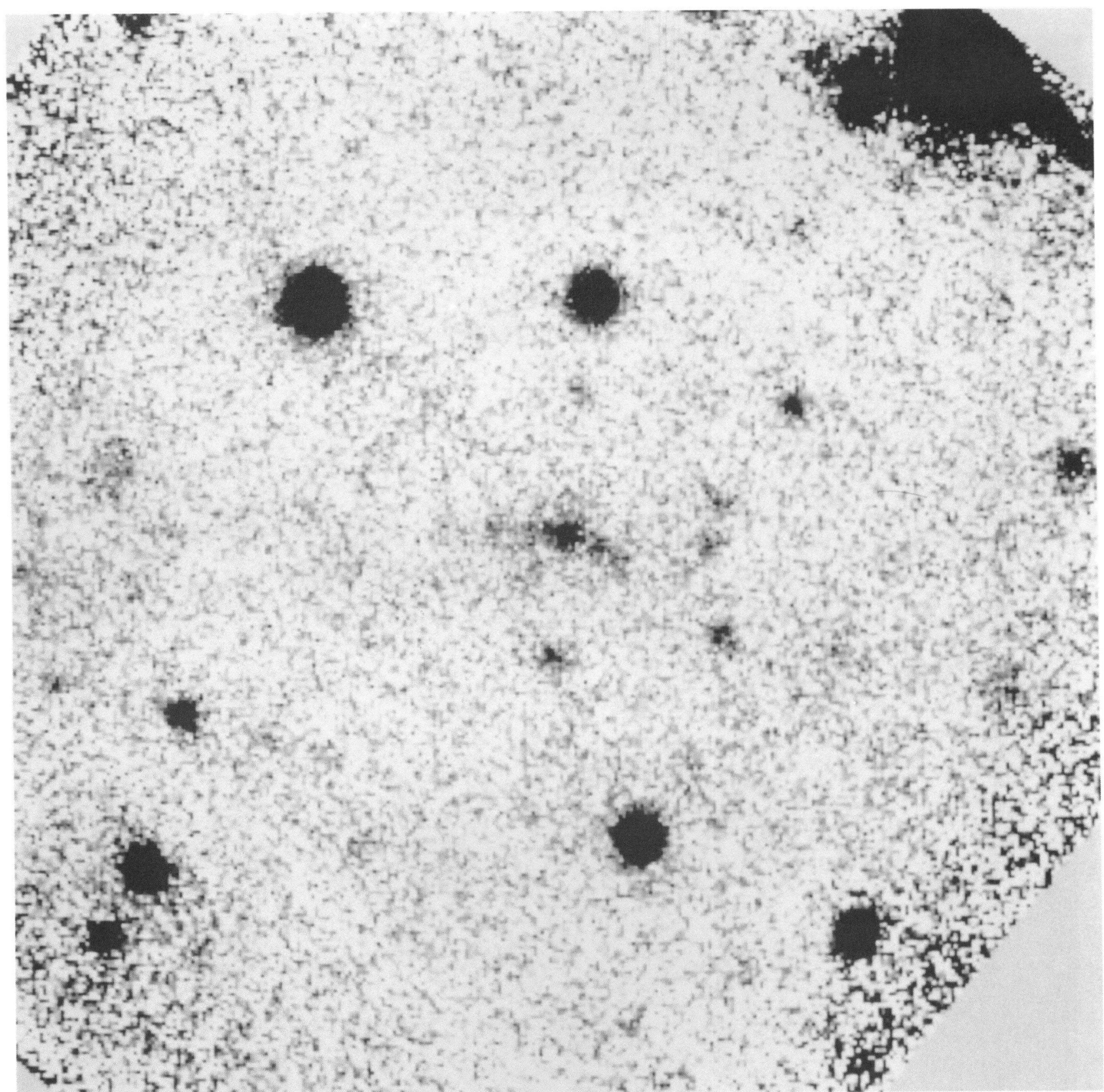

FIG. 3.-Linear gray-scale representation of the $K_{S}$ image of $4 \mathrm{C} 41.17$. The field of view is $\sim 45^{\prime \prime}$ on a side. North is at the top, and east to the left.

Graham et al. (see 420, L5) 


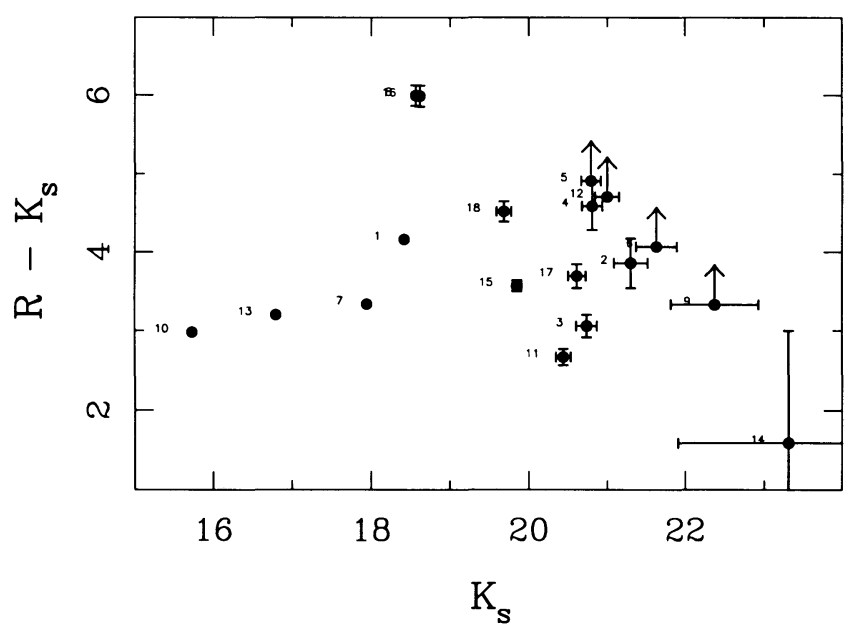

Fig. 2.-Color magnitude diagram for the field of $4 \mathrm{C} 41.17$. The number beside the symbol refers to the identification in Fig. 1.

therefore $K_{S}$ gives a good measure of the continuum. In a $4^{\prime \prime}$ diameter beam, 4C 41.17 has $K_{S}=19.6 \pm 0.6 \mathrm{mag}$, substantially brighter than the radio galaxy B2 $0902+34(K \simeq 21.5$ mag, $z=3.4$ ) (Eisenhardt \& Dickinson 1992). At $z=3.8$ the observed $K$ band corresponds to the rest frame B, and the $K_{S}$ magnitude can be converted directly into $M_{B}=-23.0 \mathrm{mag}$. An $L^{*}$ galaxy at $z=3.8$ has $K=23.1 \mathrm{mag}$ (if $M_{B}^{*}=-19.7$ mag), so all the faint objects in the vicinity of $4 \mathrm{C} 41.17$ are more luminous than $L^{*}$ if they are at the same distance.
TABLE 1

PHOTOMETRY ${ }^{\mathrm{a}}$

\begin{tabular}{|c|c|c|c|c|c|c|}
\hline Object & $K$ & Error & $K_{s}$ & Error & $R-K_{s}$ & Error \\
\hline $1 .$. & 18.08 & 0.04 & 18.43 & 0.03 & 4.16 & 0.04 \\
\hline $2 .$. & 21.14 & 0.31 & 21.30 & 0.22 & 3.86 & 0.32 \\
\hline $3 \ldots \ldots \ldots$ & 20.65 & 0.26 & 20.73 & 0.13 & 3.06 & 0.14 \\
\hline $4 \ldots \ldots \ldots$ & 20.80 & 0.22 & 20.80 & 0.13 & 4.59 & 0.30 \\
\hline $5 \ldots \ldots \ldots$ & 21.45 & 0.37 & 20.79 & 0.12 & 4.91 & $\ldots$ \\
\hline $6 \ldots \ldots \ldots$ & 21.17 & 0.28 & 21.63 & 0.26 & 4.07 & \\
\hline $7 \ldots \ldots \ldots$ & 17.80 & 0.01 & 17.95 & 0.01 & 3.33 & 0.01 \\
\hline $8 \ldots \ldots \ldots$ & 18.41 & 0.03 & 18.57 & 0.02 & 6.00 & 0.13 \\
\hline $9 \ldots$ & 22.29 & 0.78 & 22.37 & 0.55 & 3.33 & \\
\hline$\ldots \ldots$ & 15.54 & 0.00 & 15.73 & 0.00 & 2.98 & 0.00 \\
\hline $11^{\mathrm{b}}$. & 19.06 & 0.04 & 20.44 & 0.09 & 2.67 & 0.10 \\
\hline $12 \ldots$ & 20.83 & 0.17 & 20.99 & 0.15 & 4.71 & \\
\hline $13 \ldots \ldots \ldots$ & 16.67 & 0.00 & 16.80 & 0.00 & 3.20 & 0.01 \\
\hline $14 \ldots \ldots \ldots$ & 21.63 & 0.37 & 23.31 & 1.40 & 1.59 & 1.41 \\
\hline $15 \ldots \ldots \ldots$ & 19.78 & 0.07 & 19.85 & 0.06 & 3.57 & 0.07 \\
\hline $16 \ldots$ & 18.41 & 0.02 & 18.62 & 0.03 & 5.99 & 0.13 \\
\hline $17 \ldots \ldots \ldots$ & 21.05 & 0.22 & 20.61 & 0.11 & 3.69 & 0.15 \\
\hline $18 \ldots \ldots \ldots$ & 19.46 & 0.06 & 19.68 & 0.09 & 4.52 & 0.13 \\
\hline
\end{tabular}

a In a 3" circular aperture. Errors include only the statistical uncertainty. The absence of an entry in the error column indicates a $3 \sigma$ upper limit.

${ }^{b}$ Nucleus of $4 \mathrm{C} 41.17$.

At $K$ (Figs. 4 and 5 [Plate L4]) the nuclear region of $4 \mathrm{C}$ 41.17 appears as a double source separated by 0.65 . The two $K$ peaks straddle the $K_{S}$ nucleus. The difference between the $K$ and $K_{S}$ morphology is due to redshifted $\mathrm{H} \beta$ and [O III] present in the $K$ filter band pass. The distribution of $K$ light, like $K_{S}$, is clumpy, and the two bright knots in the main body of the

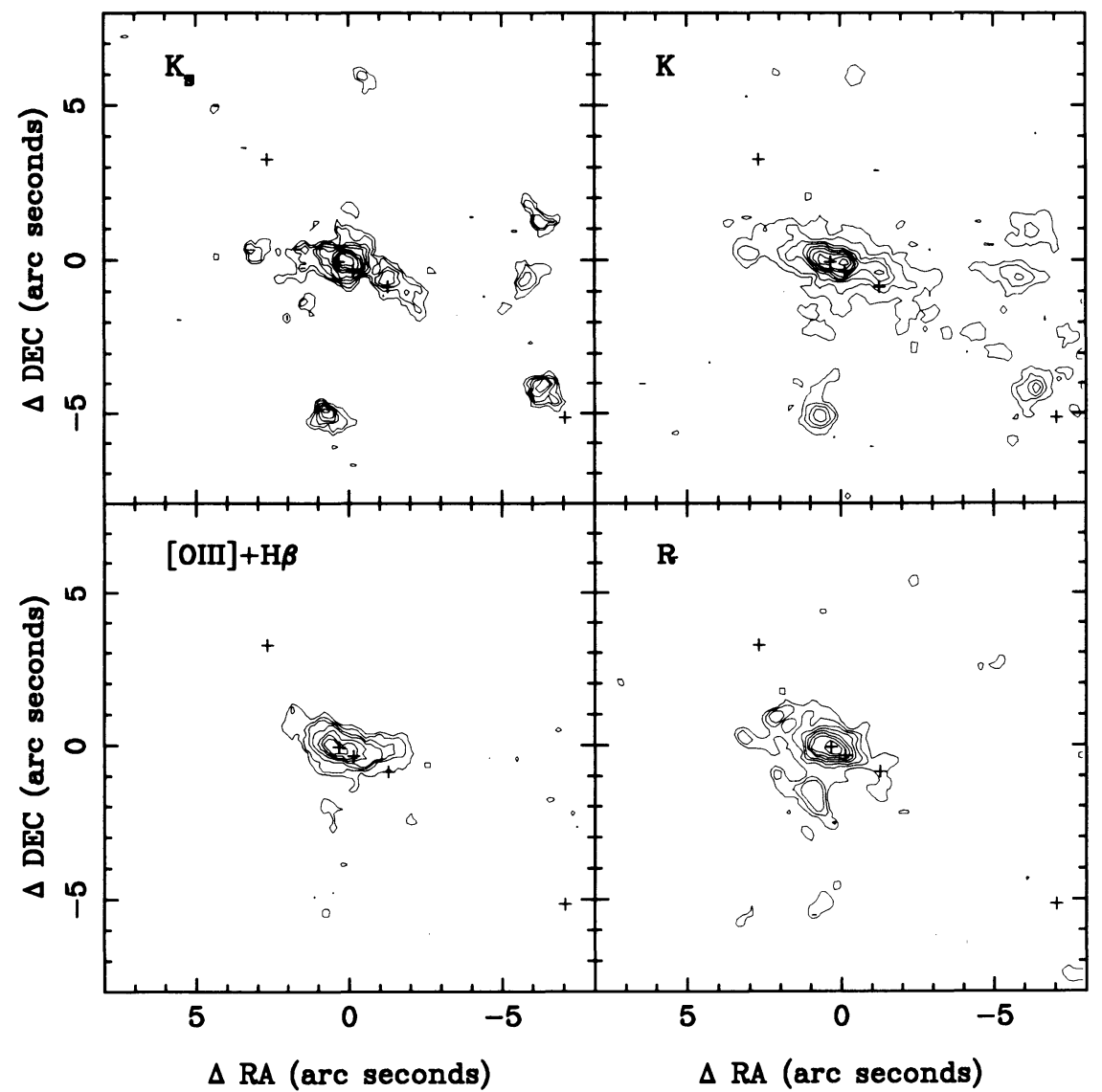

FIG. 4. Contour plots of $K_{S}, K$, and [O $\left.\mathrm{III}\right]+\mathrm{H} \beta$ and $R$ images of $4 \mathrm{C} 41.17$. The positions of the main radio components, $\mathrm{A}, \mathrm{B}_{1}, \mathrm{~B}_{3}$, and $\mathrm{C}$ (from west to east), as identified by Chambers et al. (1990), are shown as crosses. 


\section{PLATE L4}

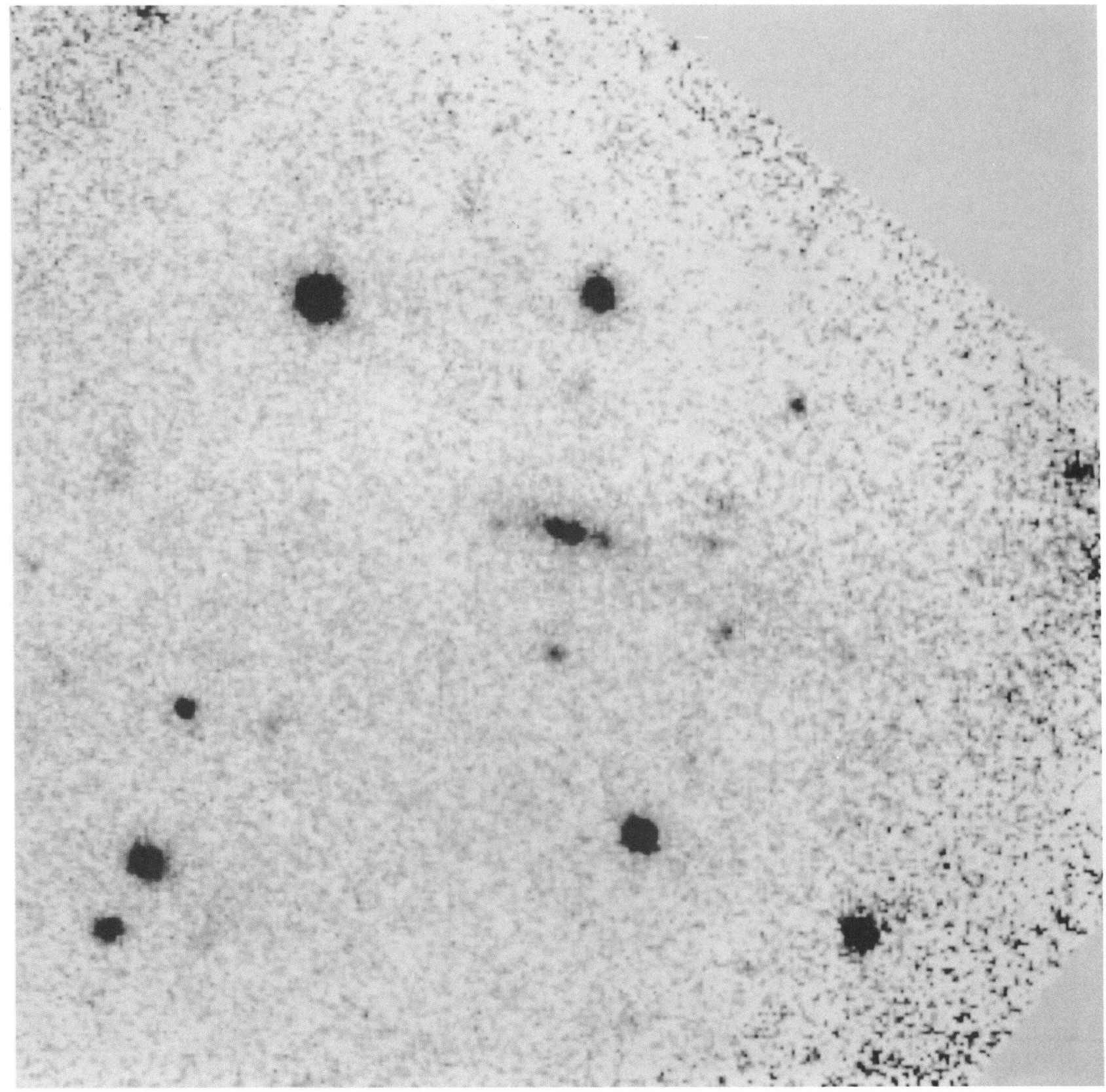

Fig. 5. - Linear gray-scale representation of the $K$ image of $4 \mathrm{C} 41.17$. The field of view is $\sim 45^{\prime \prime}$ on a side. North is at the top, and east to the left.

Graham et al. (see 420, L6) 
galaxy, seen in the $K_{S}$ image, are also present at $K$. All the faint companions also appear in the $K$ image, and low surface brightness emission runs from the main body of the galaxy along the radio axis toward radio component $A$, and to the south. Figure 6 [Plate L5] shows the $R$-band image.

A $\mathrm{H} \beta+[\mathrm{O}$ III] line emission image can be constructed by subtracting the $K_{S}$ image from $K$. The resultant image (Fig. 4) has a band pass of 2.3-2.4 $\mu \mathrm{m}$. Before subtraction the $K$ and $K_{S}$ images were smoothed to the same spatial resolution. The resultant image shows a comma-shaped emission-line knot. Although the nebula is generally aligned along the radio axis, it shows a distinct curvature away from the radio axis toward an east-west direction, reminiscent of the $\operatorname{Ly} \alpha$ halo. Unlike the original $K$ image the $\mathrm{H} \beta+[\mathrm{O} \mathrm{III}]$ image shows a single peak. Because the $K$ image was smoothed before subtraction, the resolution of the $\mathrm{H} \beta+[\mathrm{O}$ III $]$ image is too low to resolve these peaks, but the line emission image still suggests a double structure. The best-seeing Ly $\alpha$ image of Chambers et al. (1990) has a resolution of 1".0 FWHM and shows only a single peak. None of the weaker knots in the body of the galaxy, or any of the nearby companion sources are detected in the line emission image. The faint emission which runs along the radio axis at $K$ is just visible in the $\mathrm{H} \beta+[\mathrm{O} \mathrm{III}]$ image. This emission is harder to discern because of the additional $\simeq 2^{1 / 2}$ noise in the difference image.

\subsection{Comparison with Radio and Optical}

None of the infrared sources is precisely coincident with a radio source - and it might be fortuitous if one was, due to the uncertainty between optical and radio reference frames-but there is a clear association and alignment between radio and infrared emission. High-redshift $(0.6<z<2)$ radio galaxies exhibit elongated optical emission which is closely aligned with the axes defined by their radio lobes (McCarthy et al. 1987; Chambers et al. 1987). Since the $K_{s}$ band samples the rest frame $B$, the preferential orientation of the $2 \mu \mathrm{m}$ light along the radio axis shows that, even at the highest redshifts, the alignment effect is present in the rest frame visible light. This suggests that common mechanisms determine the morphology of radio galaxies between $z \simeq 0.6$ and $z \simeq 4$. Comparison of the $K, K_{S}$, and $R$ images suggests that the alignment of emission along the radio axis is most pronounced at $2 \mu \mathrm{m}$. Compared to the elongated $K_{S}$ image, the $R$-band emission is amorphous.

The positions of the main radio components, $A, B_{1}, B_{2}, B_{3}$, and $C$, as identified by Chambers et al. (1990), are shown as crosses on contour plots (Fig. 4). The $K_{s}$ peak lies close $(0 " .3$ west) to radio peak $\mathrm{B}_{3}$. The two $K$ peaks straddle $\mathrm{B}_{3}$, and the western $K$ peak is only slightly offset from the weak radio core $B_{2}$. Source $B_{1}$ lies in the arc of emission that defines the body of the galaxy, and is next to a clump present in the $K_{S}$ and $K$ images. One newly detected infrared companion (source 5 ) is close to radio component $\mathrm{A}\left(0.8\right.$ east, $1^{\prime \prime} .0$ north) and falls along the axis defined by the radio sources. No infrared counterpart of radio source $\mathrm{C}$ is detected.

Comparison of the $K$ and $K_{S}$ images with the Hubble Space Telescope image of 4C 41.17 by Miley et al. (1992) in the F702W filter reveals close similarities. The $K_{s}$ peak is coincident with the $H S T$ component $\mathrm{H}_{4}$. The $K_{S}$ nuclear contours are extended along the position angle joining $\mathrm{H}_{2}$ and $\mathrm{H}_{4}$, suggesting that a second peak, at the location of $\mathrm{H}_{2}$, might be resolved at $K_{S}$ in better seeing. The western $K$ nucleus is accurately aligned with feature $\mathrm{H}_{2}$. The eastern nucleus does not correspond to a HST peak, but to resolved emission that extends to the east. The two weaker $K_{S}$ and $K$ sources in the main body of the galaxy also align with structure seen in the $H S T$ image: the $2 \mu \mathrm{m}$ peak $1^{\prime \prime}$ west of the $K_{S}$ nucleus lines up with $\mathrm{H}_{1}$, while the $2 \mu \mathrm{m}$ source $3^{\prime \prime}$ east of the nucleus appears in the HST image (this source is outside of the field of view displayed by Miley et al. 1993).

If we shift the radio reference frame 0.3 north relative to the optical one, so that $\mathrm{B}_{2}$ and $\mathrm{B}_{3}$ coincide with $\mathrm{H}_{2}$ and $\mathrm{H}_{4}$ (cf. Miley et al. 1992) then the correlation between the radio and infrared improves. The western $K$ nucleus now lines up accurately with $B_{2}$, and the alignment with $B_{1}$ is closer.

Observations of $4 \mathrm{C} 41.17$ reveal a remarkable small-scale $(\sim 500 \mathrm{pc})$ correlation between emission seen in the rest frame UV, blue, and radio. This correlation suggests that it is unlikely that the emission is scattered light from beamed nuclear emission. The clear curvature of the $K_{S}$ image reinforces this conclusion. There is good correlation between the radio, optical, and infrared, but the correlation is not complete. There is no radio source associated with the knot of emission $3^{\prime \prime}$ east of the nucleus, and no optical or infrared emission associated with radio source $\mathrm{C}$. This may provide some difficulties for the inverse Compton model (Daly 1992).

\subsection{Contribution of Line Emission}

The large difference between $K$ and $K_{s}, K_{s}-K=0.9$ $\pm 0.06 \mathrm{mag}$ shows that the $2 \mu \mathrm{m}$ light is severely contaminated by bright emission lines. This implies that $64 \pm 6 \%$ of the $K$ light measured in a 4 " diameter beam is due to $\overline{\mathrm{H}} \beta$ and [O III]. A significant fraction of the $K$-band light is due to line emission-much larger than the $15 \%$ measured by Eales \& Rawlings (1993). The Eales \& Rawlings measurement relies on comparing slit spectroscopy with published photometry. If we compare Eales \& Rawlings's [O III] flux in a $3^{\prime \prime}$ diameter beam with the $K$ flux, then the fraction of [O III] is $40 \% \pm 10 \% . \mathrm{H} \beta$ probably contributes another $\simeq 10 \%$, and therefore most of the discrepancy is resolved.

4C 41.17 is similar to other high-redshift radio galaxies where the $K$-band magnitudes are significantly perturbed by line emission (Eisenhardt \& Dickinson 1992; Eales \& Rawlings 1993). The infrared magnitudes of high-redshift galaxies are vital for deciding the cosmological status of these young objects. Red optical-infrared colors would suggest the presence of large numbers of giants and imply an evolved population indicative of an early epoch of formation (Lilly 1988, 1989; Chambers \& Charlot 1990). If line emission dominates broadband magnitudes then this factor must be taken into account when interpreting the $K$ Hubble diagram (cf. Eales \& Rawlings 1993).

\subsection{Colors and Number Counts}

Figure 2 shows a color-magnitude diagram for the field of 4C 41.17. 4C 41.17 is the bluest non-stellar object in the field. The nature of the UV-optical continuum emission in radio galaxies is uncertain. The high degree of polarization suggests that scattered nuclear light may contribute to the observed continuum in some objects (Di Serego Alighieri et al. 1989; Jannuzi \& Elston 1991; Tadhunter et al. 1992). Spinrad et al. (1993) report a strong Lyman continuum break in 4C 41.17, which is good evidence for a stellar origin for the UV light. If the $R$ and $K_{S}$ band light is starlight, is the blue color evidence that $4 \mathrm{C} 41.17$ is a primeval galaxy forming its first generation of stars? For $4 \mathrm{C} 41.17, R-K_{S}=2.7 \pm 0.1 \mathrm{mag}$ in a $3^{\prime \prime}$ diameter beam. Eisenhardt \& Dickinson (1992) argued that B2 
PLATE L5

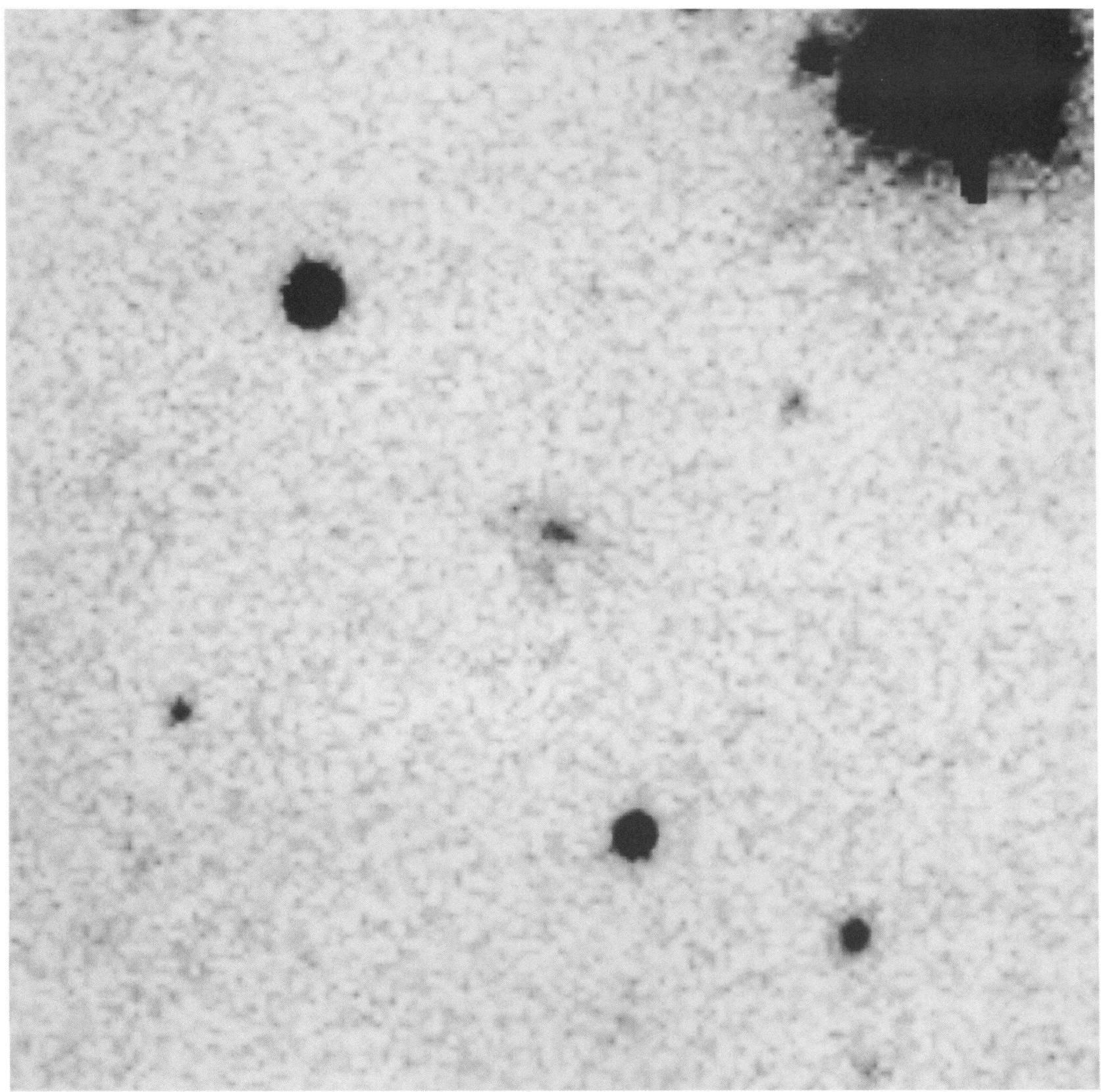

FIG. 6.-Linear gray scale representation of the $R$ image of $4 \mathrm{C} 41.17$. The field of view is $\sim 45^{\prime \prime}$ on a side. North is at the top, and east to the left. Graham et al. (see 420, L7) 
$0902+34$, with $R-K=1.9_{-\infty}^{+1.1}$, has a spectrum characteristic of a protogalaxy. For comparison we have calculated the colors of galaxies redshifted to $z=3.8$, using the model spectral energy distributions of Bruzual \& Charlot (1993). These yield $R-K_{S}=7.33,3.95,3.07$, and $2.21 \mathrm{mag}$ for $\mathrm{E}, \mathrm{Sb}$, Sc, and Im galaxies, respectively. The $R-K_{S}$ color of $4 \mathrm{C} 41.17$ falls between the Sc and Im. The $R-K_{S}$ color of $4 \mathrm{C} 41.17$ is therefore characteristic of a stellar population which has recent or ongoing star formation. Since the $R$-band corresponds to rest frame ultraviolet, the reddening correction to the observed $R-K_{S}$ color is large, $1.8 A_{V}$ (Matthis 1990). Consequently even a small amount of dust can significantly effect the observed color.

To place limits on the age of $4 \mathrm{C} 41.17$ we have calculated the colors of stellar populations formed in an instantaneous burst and by continuous star formation using the galaxy isochrone synthesis library of Bruzual \& Charlot (1993). The models assume a Salpeter initial mass function between 0.1 and 125 $M_{\odot}$ and solar metallicity and no reddening. Comparison of the calculated colors as a function of time with the observed colors places two extreme limits on the age. The instantaneous starburst matches the observed color after $0.07 \mathrm{Gyr}$, while continuous star formation must proceed for $10 \mathrm{Gyr}$ to achieve the observed color. In consequence, the limits on the age of $4 \mathrm{C}$ 41.17 are very poor, and it is premature to draw conclusions about the age of the stellar population of $4 \mathrm{C} 41.17$.

Object 3 is the next bluest object, after 4C 41.17, with $R-K_{S}=3.1 \mathrm{mag}$, and only 14,15 , and 17 have $R-K_{S}<4$ mag. The closest companions of $4 \mathrm{C} 41.17$ (4-6, 9, and 12) are distinctly red. For example, object 5 , the source adjacent to radio component $A$, and therefore most likely at the same redshift as $4 \mathrm{C} 41.17$, has $R-K_{S}>5 \mathrm{mag}$. The emission from these sources is most likely starlight, since, with the exception of object 5 , they are not coincident with radio sources, or aligned along the radio jet. Comparison with the colors of an instantaneous star burst indicates a lower limit on the age of about 0.5 Gyr. If the faint companions of $4 \mathrm{C} 41.17$ are at $z=3.8$ then their inferred age implies formation at $z \gtrsim 8$.

The color magnitude diagram for the field of 4C 41.17 (Fig. 2) contains two very red galaxies (objects 8 and 16). Both have $K=18.41 \pm 0.03 \mathrm{mag}$ and $R-K=6.16 \pm 0.13 \mathrm{mag}$ and $6.20 \pm 0.13 \mathrm{mag}$, respectively. Both objects are galaxies since they are resolved with a FWHM $\simeq 0$. 5 . The colors are those of an unevolved elliptical galaxy at $z \simeq 1.1$. Normal spirals galaxies never exhibit such red colors. The presence of two such galaxies suggests that this is not a coincidence, and that we may be viewing 4C 41.17 through a foreground cluster. If $M_{B}^{*}=-19.7 \mathrm{mag}$, then at $z \simeq 1.1$, these galaxies correspond to $\simeq 1.6 L^{*}$, and therefore would rank among the brighter cluster members. Inspection of the number vs. magnitude counts shows an excess of galaxies in the magnitude range 18-19 compared with the counts of Cowie et al. (1993). This observation is consistent with the suggestion that catalogs of the most distant radio galaxies are affected by selection effects due to gravitational lensing and amplification (Hammer \& Le Fèvre 1990).

The W. M. Keck Observatory is a scientific partnership between the University of California and the California Institute of Technology, made possible by the generous gift of the W. M. Keck Foundation and support of its president, Howard Keck. We are most grateful for their visionary endowment that has made possible the first of the next generation of telescopes. It is a pleasure to thank E. Stone, W. Frazier, W. Sargent, S. Faber, E. Romano, and W. Lupton and all of the many devoted people whose unflagging efforts have made possible the success of the W. M. Keck Observatory.

We thank M. Dickinson and H. Spinrad for permission to use their $R$-band image of $4 \mathrm{C} 41.17$. Thanks are also due to $M$. Dickinson for advice and practical help on data reduction and valuable discussions on the nature of high-redshift radio galaxies.

\section{REFERENCES}

Bruzual, G., \& Charlot, S. 1993, ApJ, 405, 538

Casali, M. M., \& Hawarden, T. G. 1992, JCMT-UKIRT Newsletter, 4, 33

Chambers, K. C., Miley, G. K., \& van Breugel, W. J. M. 1987, Nature, 329, 604 . 1990, ApJ, 363, 21

Cowie, L. L., Gardner, J. P., Hu, E. M., Wainscoat, R. J., \& Hodapp, K. W. 1993, ApJ, submitted

Daly, R. A. 1992, ApJ, 399, 426

Dickinson, M., \& Spinrad, H. 1993, private communication

Di Serego Alighieri, S., Fosbury, R. A. E., Quinn, P. J., \& Tadhunter, C. N. 1989, Nature, 341, 307

Eales, S. A., \& Rawlings, S. 1993, ApJ, 411, 67

Eisenhardt, P., \& Dickinson, M. 1992, ApJ, 399, L47

Hammer, F., \& Le Fèvre, O. 1990, ApJ, 357, 38

Jannuzi, B. T., \& Elston, R. 1991, ApJ, 366, L69
Lilly, S. J. 1988, ApJ, 333, 161

Mathis, J. S. 1990, ARȦ\&A, 28, 37

Matthews, K., et al. 1994, in Proc. UCLA Conf. on Infrared Detector Arrays, ed. I. McLean \& G. Brims (Dordrecht: Kluwer), in press

McCarthy, P. J., van Breugel, W. J. M., Spinrad, H., \& Djorgovski, S. G. 1987, ApJ, 321, L29

Miley, G. K., Chambers, K. C., van Breugel, W. J. M., \& Macchetto, F. 1992, ApJ, 401, L69

Spinrad, H., Dickinson, M., Schlegel, D., \& Gonzalez, R. 1993, in Observational Cosmology, ed. G. Chincarini, T. Maccacaro, \& D. Maccagni, in press

Tadhunter, C. N., Scarrott, S. M., Draper, P., \& Rolph, C. 1992, MNRAS, 256, $53 \mathrm{p}$ 